\title{
Nonlinear Evolution Equations and Its Application to a Tumour Invasion Model
}

\author{
Akisato Kubo', Yuto Miyata², Hidetoshi Kobayashi³, Hiroki Hoshino', Naoki Hayashi' \\ ${ }^{1}$ Department of Mathematics, School of Health Sciences, Fujita Health University, Toyoake, Japan \\ ${ }^{2}$ Medical Radiation Sciences, Graduate School of Health Sciences, Fujita Health University, Toyoake, Japan \\ ${ }^{3}$ Department of Radiation Oncology, School of Medicine, Fujita Health University, Toyoake, Japan \\ ${ }^{4}$ Faculty of Radiological Technology, School of Health Sciences, Fujita Health University, Toyoake, Japan \\ Email: akikubo@fujita-hu.ac.jp,82015305@fujita-hu.ac.jp,cnc07519@yahoo.co.jp, hhoshino@fujita-hu.ac.jp,hayashi@fujita-hu.ac.jp
}

How to cite this paper: Kubo, A., Miyata, Y., Kobayashi, H., Hoshino, H. and Hayashi, N. (2016) Nonlinear Evolution Equations and Its Application to a Tumour Invasion Model. Advances in Pure Mathematics, 6, 878-893.

http://dx.doi.org/10.4236/apm.2016.612066

Received: September 12, 2016

Accepted: November 14, 2016

Published: November 17, 2016

Copyright $\odot 2016$ by authors and Scientific Research Publishing Inc. This work is licensed under the Creative Commons Attribution International License (CC BY 4.0).

http://creativecommons.org/licenses/by/4.0/

\begin{abstract}
We consider nonlinear evolution equations with logistic term satisfying initial Neumann-boundary condition and show global existence in time of solutions to the problem in arbitrary space dimension by using the method of energy. Applying the result to a mathematical model of tumour invasion, we discuss the property of the rigorous solution to the model. Finally we will show the time depending relationship and interaction between tumour cells, the surrounding tissue and matrix degradation enzymes in the model by computer simulations. It is seen that our mathematical result of the existence and asymptotic behaviour of solutions verifies our simulations, which also confirm the mathematical result visibly.
\end{abstract}

\section{Keywords}

Nonlinear Evolution Equation, Mathematical Analysis, Tumour Invasion, Proliferation, Re-Establishment

\section{Introduction}

In this paper we consider the initial Neumann-boundary value problem of nonlinear evolution equations with logistic term, arising from tumour invasion models with proliferation and re-establishment: (NE)

$$
\begin{cases}\partial_{t}^{2} u=D \Delta u_{t}+\nabla \cdot\left(\chi\left(u_{t}, \mathrm{e}^{-u}\right) \mathrm{e}^{-u} \nabla u\right)+\mu\left(1-u_{t}\right) u_{t} & \text { in } \Omega \times(0, T) \\ \left.\frac{\partial}{\partial v} u\right|_{\partial \Omega}=0 & \text { on } \partial \Omega \times(0, T) \\ u(x, 0)=u_{0}(x), u_{t}(x, 0)=u_{1}(x) & \text { in } \Omega\end{cases}
$$




$$
\begin{aligned}
& \frac{\partial}{\partial t}=\partial_{t}, \frac{\partial}{\partial x_{i}}=\partial_{x_{i}}, i=1, \cdots, n, \quad \nabla u=\left(\partial_{x_{1}} u, \cdots, \partial_{x_{n}} u\right) \\
& \nabla^{2} u=\nabla \cdot \nabla u=\Delta u=\partial_{x_{1}}^{2} u+\cdots+\partial_{x_{n}}^{2} u
\end{aligned}
$$

where $u:=u(x, t)$ for $(x, t) \in \Omega \times(0, T), D$ and $\mu$ are positive constants, $\Omega$ is a bounded domain in $R^{n}$ and $\partial \Omega$ is a smooth boundary of $\Omega$ and $v$ is the outer unit normal vector.

Let us introduce function spaces used in below. First, $H^{l}(\Omega)$ denotes the usual Sobolev space $W^{l, 2}(\Omega)$ of order $l$ on $\Omega$. For functions $h(x, t)$ and $k(x, t)$ defined in $\Omega \times[0, \infty)$, we denote

$$
(h, k)(t)=\int_{\Omega} h(x, t) k(x, t) \mathrm{d} x,\|h\|^{2}(t)=(h, h)(t),\|h\|_{l}^{2}(t)=\sum_{|\beta| \leq l}\left\|\partial_{x}^{\beta} h(\cdot, t)\right\|^{2}(t)
$$

where $\beta$ is a multi-index for $\beta=\left(\beta_{1}, \cdots, \beta_{n}\right)$.

The eigenvalues of $-\Delta$ with the homogeneous Neumann boundary conditions are denoted by $\left\{\lambda_{i} \mid i=0,1,2, \cdots\right\}$ satisfying $0=\lambda_{0}<\lambda_{1} \leq \cdots \rightarrow+\infty$ and $\varphi_{i}=\varphi_{i}(x)$ indicates the $L^{2}$ normalized eigenfunction corresponding to $\lambda_{i}$. For a non-negative integer $l$, we set $W^{l}(\Omega)$ as a closure of $\left\{\varphi_{1}, \varphi_{2}, \cdots, \varphi_{n}, \cdots\right\}$ in the function space $H^{l}(\Omega)$. It is noticed that we have $\int_{\Omega} h(x) \mathrm{d} x=0$ for $h(x) \in W^{l}(\Omega)$, which enables us to use Poincare's Inequality.

Putting $u(x, t)=a+b t+v(x, t)$ in (1) it follows from (1) that

$$
\partial_{t}^{2} v=D \Delta v_{t}+\nabla \cdot\left(\chi\left(b+v_{t}, \mathrm{e}^{-a-b t-v}\right) \mathrm{e}^{-a-b t-v} \nabla v\right)+\mu v_{t}(t)\left(1-2 b-v_{t}\right)+\mu b(1-b)
$$

where $a$ and $b$ are positive parameters. (NE) is rewritten by the following problem.

$$
\text { (RP) }\left\{\begin{array}{l}
Q[v]=\partial_{t}^{2} v-D \Delta v_{t}-\nabla \cdot\left(\chi^{a, b}(v) \mathrm{e}^{-a-b t-v} \nabla v\right)-\mu v_{t}\left(1-2 b-v_{t}\right)-\mu b(1-b)=0, \\
\left.\frac{\partial}{\partial v} v\right|_{\partial \Omega}=0, \\
v(x, 0)=v_{0}(x), v_{t}(x, 0)=v_{1}(x) .
\end{array}\right.
$$

where $\chi^{a, b}(v)=\chi\left(b+v_{t}, \mathrm{e}^{-a-b t-v}\right)$. We will show the global existence in time of solutions of (RP), which gives our desired result of (NE).

Applying the above result to the following mathematical model of tumour invasion proposed by Chaplain and Lolas [1], we have a rigorous mathematical understanding to tumour invasion for the key variables $n, m$ and $f$.

$$
(\mathrm{C}-\mathrm{L})\left\{\begin{array}{l}
\frac{\partial n}{\partial t}=d_{n} \Delta n-x_{n} \nabla \cdot(n \nabla m)-\gamma \nabla \cdot(n \nabla f)+\mu_{1} n(1-n-f) \\
\frac{\partial f}{\partial t}=-\eta m f+\mu_{2} f(1-n-f) \\
\frac{\partial m}{\partial t}=d_{m} \Delta m+\alpha n-\beta m
\end{array}\right.
$$

where $n:=n(x, t)$ is the density of tumour cells, $m:=m(x, t)$ is matrix degradation enzymes (MDEs) concentration and $f:=f(x, t)$ is extracellular matrix (ECM) density in $\Omega \times(0, T)$ and $d_{n}, \gamma, \mu_{1}, \eta, \mu_{2}, d_{m}, \alpha$ and $\beta$ are positive constants. 
In the right hand side of (4) the second, third and fourth terms mean chemotaxis, haptotaxis and proliferation of tumour cell respectively. Also the second term of the right hand side of (5) describes the re-establishment of ECM. We consider an initial boundary value problem for $(\mathrm{C}-\mathrm{L})$ satisfying

$$
\begin{aligned}
& \frac{\partial n}{\partial v}=\frac{\partial f}{\partial v}=\frac{\partial m}{\partial v}=0, \text { on } \partial \Omega \times(0, \infty), \\
& n(x, 0)=n_{0}(x), f(x, 0)=f_{0}(x), m(x, 0)=m_{0}(x),
\end{aligned}
$$

where $\left\{n_{0}(x), f_{0}(x), m_{0}(x)\right\}$ are initial data of $\{n(x, t), f(x, t), m(x, t)\}$.

Chaplain and Anderson [2], corresponding to the case of $x_{n}=\mu_{1}=\mu_{2}=0$ in (C-L), proposed a mathematical model on generic solid tumour growth at the avascular stage. While most tumours are asymptomatic at this stage, it is still possible for cells to escape and migrate to the lymph nodes and for more aggressive tumours to invade.

In our previous papers [3] [4], we consider only the case of $\mu_{2}=0$ and $x_{n}=0$ for our convenience. In this paper we may consider the case where $x_{n}$ is sufficiently small positive constant. Actually compared with the effect of haptotaxis, the one of chemotaxis is very small. Furthermore we study the case of $\mu_{1}, \mu_{2}>0$, which describes tumour invasion phenomena with tumour cell proliferation and re-establishment of ECM respectively.

In the final section by computer simulations of our model (by Mathematica 8) we can easily observe time-dependent interaction and the relationship between the above components in complicated procedure of tumour invasion and a comparison to our rigorous mathematical result. Comparing our mathematical result with computer simulations we will gain a better understanding of the mechanism of tumour invasion.

\section{Existence Theorem of (NE)}

By deriving the energy estimate of (RP) (see [3]-[9]) and considering the iteration scheme we obtain existence of solutions to (RP) by the standard argument to show the convergence of solutions of the iteration scheme.

In the same way as used in [3] [4] [5] [6] [7] we have the following estimates of (RP). We begin with $\left(Q[v], v_{t}\right)=0$ in order to obtain a basic estimate of (RP). Then we have for $b=1$,

$$
2\left(\partial_{t}^{2} v-D \Delta v_{t}-\nabla \cdot\left(\chi^{a, b}(v) \mathrm{e}^{-a-b t-v} \nabla v\right), v_{t}\right)-2 \mu\left(\left(-1-v_{t}\right) v_{t}, v_{t}\right)
$$

by the integration by parts

$$
=\partial_{t}\left\|v_{t}\right\|^{2}+2 D\left\|\nabla v_{t}\right\|^{2}+2\left(\chi^{a, b}(v) \mathrm{e}^{-a-b t-v} \nabla v, \nabla v_{t}\right)+2 \mu\left(\left(1+v_{t}\right) v_{t}, v_{t}\right)=0 .
$$

It is noticed that the following estimate is obtained in [4] [5] [6] [7] for $0<b^{\prime}<1$,

$$
\mathrm{e}^{-a-t-u}<C \mathrm{e}^{-b^{\prime} t} .
$$

Then for the nonlinear term we have by using (8) 


$$
\begin{aligned}
\int_{0}^{t}\left(\chi^{a, b}(v) \mathrm{e}^{-a-\tau-v} \nabla v, \nabla v_{\tau}\right) \mathrm{d} \tau & =\int_{0}^{t}\left(\chi^{a, b}(v) \mathrm{e}^{-a-\tau-v} \sqrt{\varepsilon}-1 \nabla v, \sqrt{\varepsilon} \nabla v_{\tau}\right) \mathrm{d} \tau \\
& \leq C\left(\varepsilon^{-1} \int_{0}^{t}\left(\mathrm{e}^{-2 a-2 b^{\prime} \tau} \nabla v, \nabla v\right) \mathrm{d} t+\varepsilon \int_{0}^{t}\left\|\nabla v_{\tau}\right\|^{2} \mathrm{~d} \tau\right)
\end{aligned}
$$

where we used Dionne [10] for the estimate of nonlinear terms and $\varepsilon$ is sufficiently small positive constant. Therefore we have by integrating the both sides of (7) over $(0, t)$ and using (9)

$$
\begin{aligned}
& \left\|v_{t}\right\|^{2}(t)+\int_{0}^{t}\left\|\nabla v_{\tau}\right\|^{2}(\tau) \mathrm{d} \tau+\mu \int_{0}^{t}\left(\left(1+v_{\tau}\right) v_{\tau}, v_{\tau}\right) \mathrm{d} \tau \\
& \leq C E[v](0)+C\left(\varepsilon^{-1} \int_{0}^{t}\left(\mathrm{e}^{-2 a-2 b^{\prime} \tau} \nabla v, \nabla v\right) \mathrm{d} \tau+\varepsilon \int_{0}^{t}\left\|\nabla v_{\tau}\right\|^{2}(\tau) \mathrm{d} \tau\right),
\end{aligned}
$$

where we denote $E[v](t)=\left\|v_{t}\right\|^{2}(t)+\|\nabla v\|^{2}(t)$.

Since the last term of the right hand side of (10) is negligible for sufficiently small $\varepsilon$, we have by integration by parts with respect to $t$

$$
\begin{aligned}
& \left\|v_{t}\right\|^{2}(t)+\int_{0}^{t}\left\|\nabla v_{\tau}\right\|^{2}(\tau) \mathrm{d} \tau+\int_{0}^{t}\left(\left(1+v_{\tau}\right) v_{\tau}, v_{\tau}\right) \mathrm{d} \tau \\
& \leq C E[v](0)+C \mathrm{e}^{-a} \int_{0}^{t}\left\|\nabla v_{\tau}\right\|^{2}(\tau) \mathrm{d} \tau
\end{aligned}
$$

Taking a sufficiently large for the second term of the right hand side of (11) is negligible. Since $\left(1+v_{t}\right)>0$ if $\left|v_{t}\right| \ll 1$, the third term in the left hand side of (11) is positive. Then we obtain a basic energy estimate

$$
\left\|v_{t}\right\|^{2}(t)+\int_{0}^{t}\left\|\nabla v_{\tau}\right\|^{2}(\tau) \mathrm{d} \tau+\int_{0}^{t}\left\|v_{\tau}\right\|^{2}(\tau) \mathrm{d} \tau \leq C E[v](0) .
$$

Replacing $\nabla^{k} v, k \leq M$ instead of $v$ in the above procedure, we obtain the following estimate of higher order.

Lemma 1 (Energy estimate of (RP)) Assume that $\chi\left(s_{1}, s_{2}\right)$ for $\left(s_{1}, s_{2}\right) \in R^{2}$ satisfies appropriate smooth regularity condition. Supposed that $\left|v_{t}\right| \ll 1$ we have the energy estimate of $(R P)$ for $M \geq[n / 2]+3$

$$
\sum_{j=1}^{M+1}\left\{\left\|\nabla^{j-1} v_{t}\right\|^{2}(t)+\int_{0}^{t}\left\|\nabla^{j} v_{\tau}\right\|^{2}(\tau) \mathrm{d} \tau\right\} \leq C E_{M}[v](0),
$$

where we denote for any non-negative integer $k \leq M, E_{k}[v](t)=E\left[\nabla^{k} v\right]$.

We consider the iteration scheme of (RP):

$$
(i+1)\left\{\begin{array}{l}
Q_{i}\left[v_{i+1}\right]=\partial_{t}^{2} v_{i+1}-D \Delta\left(v_{i+1}\right)_{t}-\nabla \cdot\left(\chi^{a, 1}\left(v_{i}\right) \mathrm{e}^{-(a+t)-v_{i}} \nabla v_{i+1}\right)+\mu_{1}\left(v_{i+1}\right)_{t}\left(1+\left(v_{i}\right)_{t}\right)=0 \\
\left.\frac{\partial}{\partial v} v_{i+1}\right|_{\partial \Omega}=0, \\
v_{i+1}(x, 0)=v_{0}(x),\left(v_{i+1}\right)_{t}(x, 0)=v_{1}(x),
\end{array}\right.
$$

where 


$$
v_{i}=\sum_{j=1}^{\infty} f_{i j}(t) \varphi_{j}(x), v_{0}(x)=\sum_{j=1}^{\infty} h_{j} \varphi_{j}(x), v_{1}(x)=\sum_{j=1}^{\infty} h_{j}^{\prime} \varphi_{j}(x)
$$

(13) guarantees the uniformly bounded estimate of $(i+1)$ for $i=1,2, \cdots$. We determine $f_{i j}(t)$ by the solution of the following ordinary equations with initial data.

$$
\left\{\begin{array}{l}
\left(Q_{i}\left[v_{i+1}\right], \varphi_{j}\right)=0, \\
f_{i+1 j}(0)=h_{i+1},\left(f_{i+1 j}\right)_{t}(0)=h_{i+1}^{\prime} .
\end{array}\right.
$$

The local existence in time of $f_{i j}(t)$ is shown by the theory of ordinary differential equations. Therefore, by the energy estimates, the global existence in time of the solution $\left\{u_{i}\right\}$ satisfying the regularity required for Lemma 1 and justification of the limiting process are assured by the standard method. The energy estimate enables us to get the solution of $(\mathrm{RP})$ in

$$
\bigcap_{i=0}^{1} C^{i}\left([0, \infty) ; H^{m-i}(\Omega)\right), \quad m \geq M,
$$

by considering $Q_{i}\left[v_{i+1}\right]-Q_{i-1}\left[v_{i}\right]$ and standard argument of convergence for $v_{i+1}-v_{i}$ (see [3]-[9]).

Then we obtain the following result of (NE) by using the above result of (RP).

Theorem 2 Assume that $\chi\left(s_{1}, s_{2}\right)$ for $\left(s_{1}, s_{2}\right) \in R^{2}$ satisfies appropriate smooth regularity condition, initial data $\left(v_{0}(x), v_{1}(x)\right)$ are sufficiently smooth for $v_{0}(x)=u_{0}(x)-a, \quad v_{1}(x)=u_{1}(x)-1$ and that $\left\|v_{1}(x)\right\|_{m}$ is small enough. For sufficiently large $a$, there is a solution for $m \geq[n / 2]+3$

$$
u(x, t)=a+t+v(x, t) \in \bigcap_{i=0}^{1} C^{i}\left([0, \infty) ; H^{m-i}(\Omega)\right)
$$

to $(N E)$ such that it satisfies the following asymptotic behavior

$$
\lim _{t \rightarrow \infty}\left\|u_{t}(x, t)-1\right\|_{m-1}=0 \text {. }
$$

\section{Application to a Tumour Invasion Model}

In the last several decades, a number of mathematical models describing the procedure of tumour growth have been the remarkable subject of research (cf. [1] [2] [11]-[19], further references therein). Especially our main concern in this section is mathematical models of avascular tumour growth proposed by Chaplain et al. (see [1] [2]). They are considered mainly by three components in the process of tumour invasion, tumour cells, ECM (extracellular matrix) and MDEs (matrix degradation enzymes) without the effect of proliferation of tumour cell. Anderson and Chaplain [2] has been developed by Chaplain and Lolas [1] additionally considering into chemotaxis, proliferation of tumour cells and re-establishment of ECM.

Their mathematical approach to above models mainly depends on numerical analysis. In this paper first we show the rigorous mathematical result of (C-L) and then computer simulations, of which the validity is guaranteed by our mathematical result.

On the other hand, there are many mathematical models which can be found in the 
literature describing tumour angiogenesis. In [20] Levine and Sleeman applied the mathematical model of Othmer and Stevens [21] for the understanding of tumour angiogenesis, which arises in the theory of reinforced random walk. Anderson and Chaplain [12] proposed a model of tumour angiogenesis taking account of endothelial tip-cell migration. The model describes cell migration governed by three factors: diffusion, chemotaxis and haptotaxis.

Rigorous Mathematical approaches to tumour growth models have been known (see [3]-[9] [20] [21] [22] [23] [24]). Levine and Sleeman [20] and Yang, Chen and Liu [24] studied the global existence in time of solutions and blow up ones to a simplified Othmer and Stevens model. Kubo et al. [3]-[9] show the time global solution and asymptotic behavior of the solution to the mathematical models proposed by [2] [12] [20] [21] [23].

\subsection{Reduced Problem}

Following to Levine and Sleeman [20] we reduce our problem to a simpler system (see [3]-[9] [20]). It is easily seen in (5) that $f(x, t)$ is written by

$$
\partial_{t}(\log f)=-\eta m+\mu_{2}(1-n-f) .
$$

Integrating (14) over $(0, t)$ for $f(x, 0)=f_{0}(x)$

$$
f(x, t)=f_{0}(x) \cdot \mathrm{e}^{-a-\eta \int_{0}^{t} m d s+\mu_{2} \int(1-n-f) \mathrm{ds}} .
$$

Put $n=1+\tilde{n}$ and $m=b+\tilde{m}$ for a constant $b>0$. Then we have

$$
f(x, t)=f_{0}(x) \cdot \mathrm{e}^{-a-b t-\eta \int_{0}^{t} \tilde{m} \mathrm{~d} s-\mu_{2} \int_{0}^{t}(\tilde{n}+f) \mathrm{ds}}
$$

denoting $\int_{0}^{t} \tilde{n} \mathrm{~d} s=u$ and $\int_{0}^{t} \tilde{m} \mathrm{~d} s=v$

$$
=f_{0}(x) \cdot \mathrm{e}^{a-b t-\eta \nu-\mu_{2}\left(u+\int_{0}^{t} f \mathrm{~d} s\right)}
$$

Substituting $f(x, t)$ by the right hand side of (14), from (4) and (6) it follows that for $x_{n}=0$

$$
\begin{aligned}
\partial_{t}^{2} u= & d_{n} \Delta u_{t}-\gamma \nabla \cdot\left(\left(1+u_{t}\right) \nabla\left(f_{0} \cdot \mathrm{e}^{-a-b t-\eta v-\mu_{2}\left(u+\int_{0}^{t} f \mathrm{ds}\right)}\right)\right) \\
& +\mu_{1} u_{t}\left(-1-u_{t}-f_{0} \cdot \mathrm{e}^{-a-b t-\eta v-\mu_{2}\left(u+\int_{0}^{t} f \mathrm{ds}\right)}\right)-\mu_{1} \mathrm{e}^{-a-b t-\eta v-\mu_{2}\left(u+\int_{0}^{t} f \mathrm{ds}\right)}
\end{aligned}
$$

and

$$
\partial_{t}^{2} v=d_{m} \Delta v_{t}+\alpha u_{t}-\beta v_{t}
$$

The nonlinear evolution Equation (1) involves (16) and so we can apply Theorem 2 to (16).

\subsection{Existence Theorem of (C-L) with $x_{n}=0$}

The Equations (16) and (17) are essentially regarded as the same type of equation as (1). 
Hence the energy estimates of $u$ and $v$ are obtained and combining these estimates we obtain the desired estimate (cf. [3] [4] [5] [6] [7]).

Lemma 3 (Energy estimate of (C-L)) We obtain the energy inequality of the reduced problem (16) and (17) with zero-Neumann boundary condition for $m>M \geq[n / 2]+1$ and sufficiently large a

$$
\begin{aligned}
& \left\|u_{t}\right\|_{M}^{2}+\int_{0}^{t}\left\|u_{s}\right\|_{M+1}^{2} \mathrm{~d} s+\left\|v_{t}\right\|_{M}^{2}+\int_{0}^{t}\left\|v_{s}\right\|_{M+1}^{2} \mathrm{~d} s+\|f\|_{M}^{2}+\int_{0}^{t}\|f\|_{M+1}^{2} \mathrm{~d} s \\
& \leq C\left(E_{a, M}[u](0)+E_{a, M}[v](0)+E_{a, M}[f](0)\right)+C_{a}
\end{aligned}
$$

where $C_{a} \rightarrow 0$ as $a \rightarrow \infty$.

Then applying the same argument as used for Theorem 2 to the above mathematical model, we obtain global existence in time and asymptotic behaviour of the solutions to our mathematical model.

Our main result for $x_{n}=0$ is as follows.

Theorem 4 For smooth initial data $\left\{n_{0}(x), f_{0}(x), m_{0}(x)\right\}$ assume that $H^{m}(\Omega)$ norms of $u_{t}(x, 0), u_{t}(x, 0)$ and $f_{0}(x)$ are sufficiently small, then there are classical solutions of $(C-L)$ for $x_{n}=0:\{n(x, t), f(x, t), m(x, t)\}$ such that they satisfy the following asymptotic behavior for $m>[n / 2]+1$

$$
\lim _{t \rightarrow \infty}\|n(x, t)-1\|_{m-1}=0, \lim _{t \rightarrow \infty} f(x, t)=0 .
$$

Also we can deal with the case of $x_{n}>0$ as follows.

\subsection{Existence Theorem of (C-L) with $x_{n}>0$}

In $\left(Q(v), v_{t}\right)=0$ for $x_{n}>0$, especially we may consider $L^{2}$-inner product composed of the chemotaxis term and $v_{t}$

$$
-x_{n}\left(\nabla \cdot(n \nabla m), v_{t}\right)=-x_{n}\left(\nabla \cdot\left(\left(b+v_{t}\right) \nabla u\right), v_{t}\right)=x_{n}\left(\left(b+v_{t}\right) \nabla u, \nabla v_{t}\right)
$$

in the same way as in Section 2

$$
\leq C_{n}\left(\left\|v_{t}\right\|_{1}^{2}+\|u\|_{1}^{2}\right)
$$

where we can take $C_{n}$ small enough for sufficiently small $x_{n}>0$. It is noticed that compared with the effect of haptotaxis, the one of chemotaxis may be quite small. Hence since we can have the same estimate as Lemma 3 for sufficiently small $x_{n}>0$, the same result as in Theorem 4 holds for this case too.

Theorem 5 Under the same assumption as in Theorem 4 we further assume that $x_{n}>0$ is sufficiently small, then we obtain the same result as stated in Theorem 4 .

\section{Computer Simulations}

In Kolev and Zubik-Kowal [16] the same type model of (C-L) for $x_{n}=0$ was considered and they obtained numerical solutions. In this paper our advantage is to show global existence in time of rigorous mathematical smooth solutions of the model and based on it carry out computer simulations. 
In the following Figures 1-7 illustrated below we show the graphs along the time by the computer simulations of the model at $t=0,0.25,0.5,0.75$ and 1.35 by Mathematica 8 . We use the parameter values of $d_{n}=0.001, d_{m}=0.001, \gamma=0.02, \eta=10$, $\mu_{1}=0 \sim 10, \mu_{2}=0 \sim 5, \alpha=0.1, \beta=0.1$ and $x_{n}=0 \sim 0.01$ specified below.

We will observe the time dependent relationship and interaction between tumour cells (Red line), the surrounding tissue (Black line) and degradation enzymes (Green line). In the graphs below a coordinate axis of the horizontal direction indicates the spatial position and vertical direction indicates the density or concentration of each component of the model.
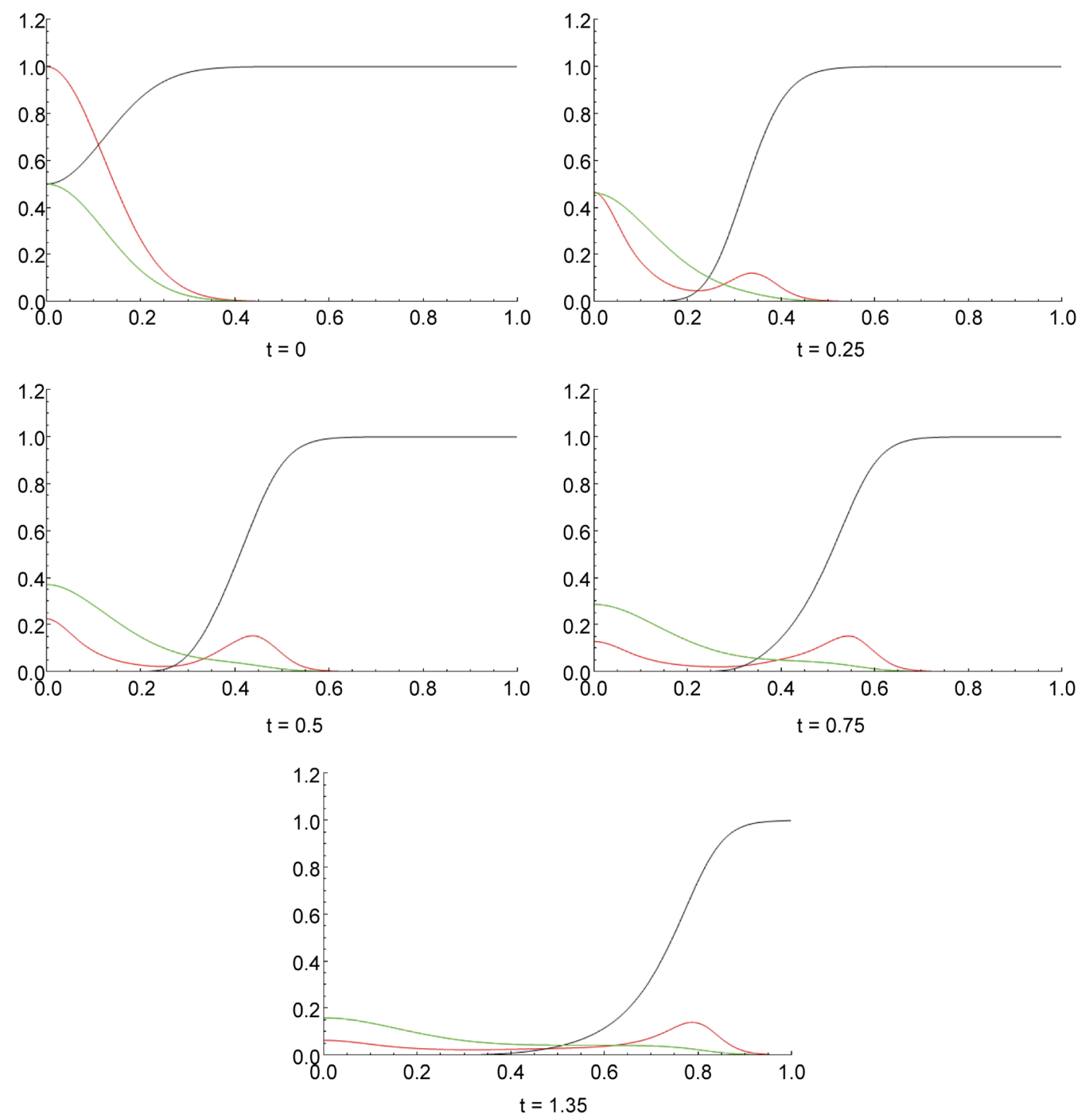

Figure 1. Interactions between the tumour and the surrounding tissue without proliferation of tumour cell, migration, and ECM re-establishment: The parameter values $d_{n}=0.001, d_{m}=0.001, \gamma=0.02, \eta=10, \mu_{1}=0$, $\mu_{2}=0, \alpha=0$ and $\beta=0.1, x_{n}=0$. We can observe that MDEs degradates the surrounding tissue, and makes space into which tumour cells move. Further tumour cells form a small peak and keep going forward inside ECM, preserving the shape. 

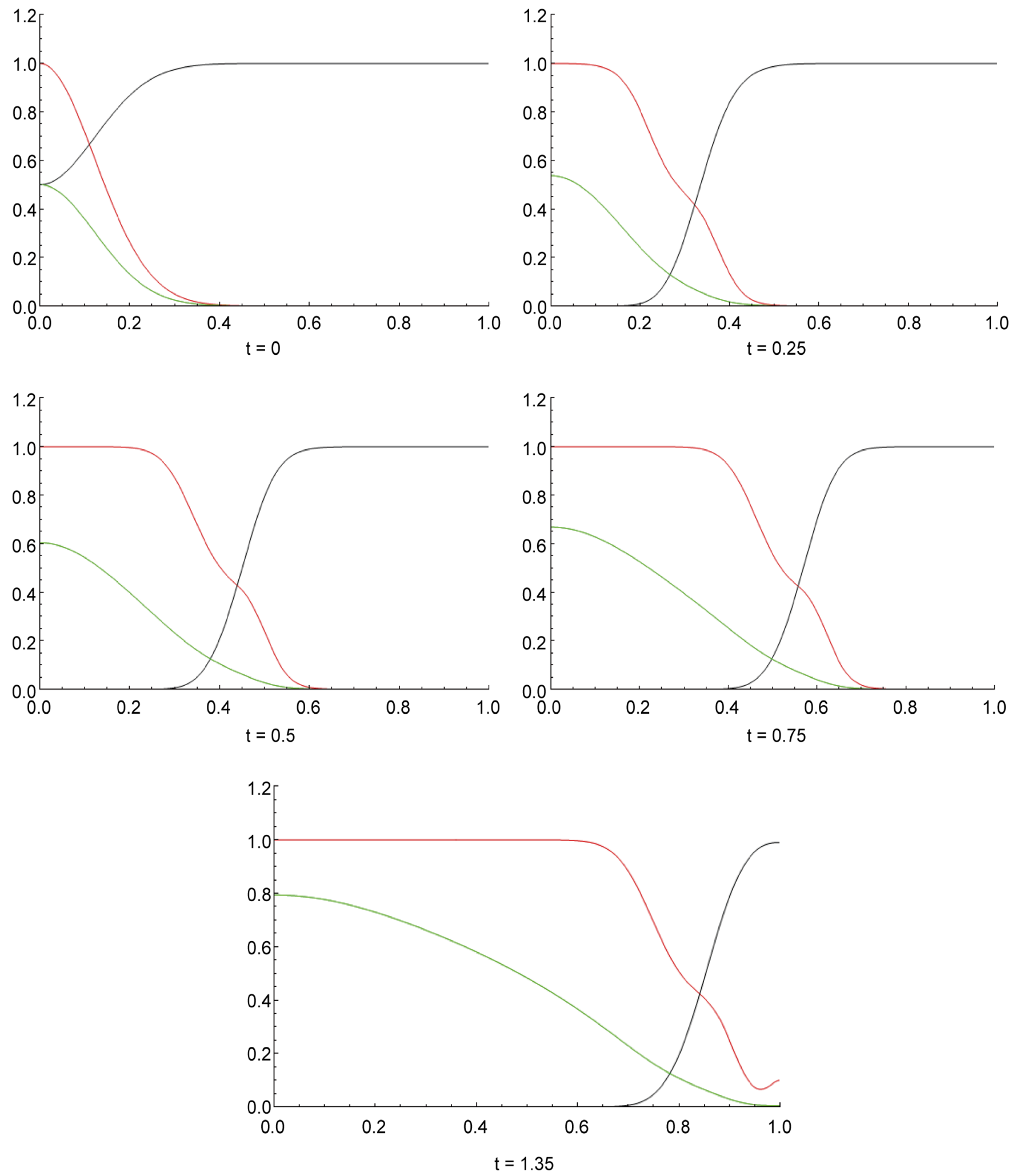

Figure 2. Tumour cell proliferation, migration, and interactions between the tumour and the surrounding tissue without ECM re-establishment: The parameter values $d_{n}=0.001, d_{m}=0.001, \gamma=0.02, \eta=10, \mu_{1}=5, \mu_{2}=0, \alpha=0.1$ and $\beta=0.1, x_{n}=0$. It is seen that tumour cell density becomes much higher than in Figure 1 inside ECM and maintains the upper bound of the logistic curve constantly. 

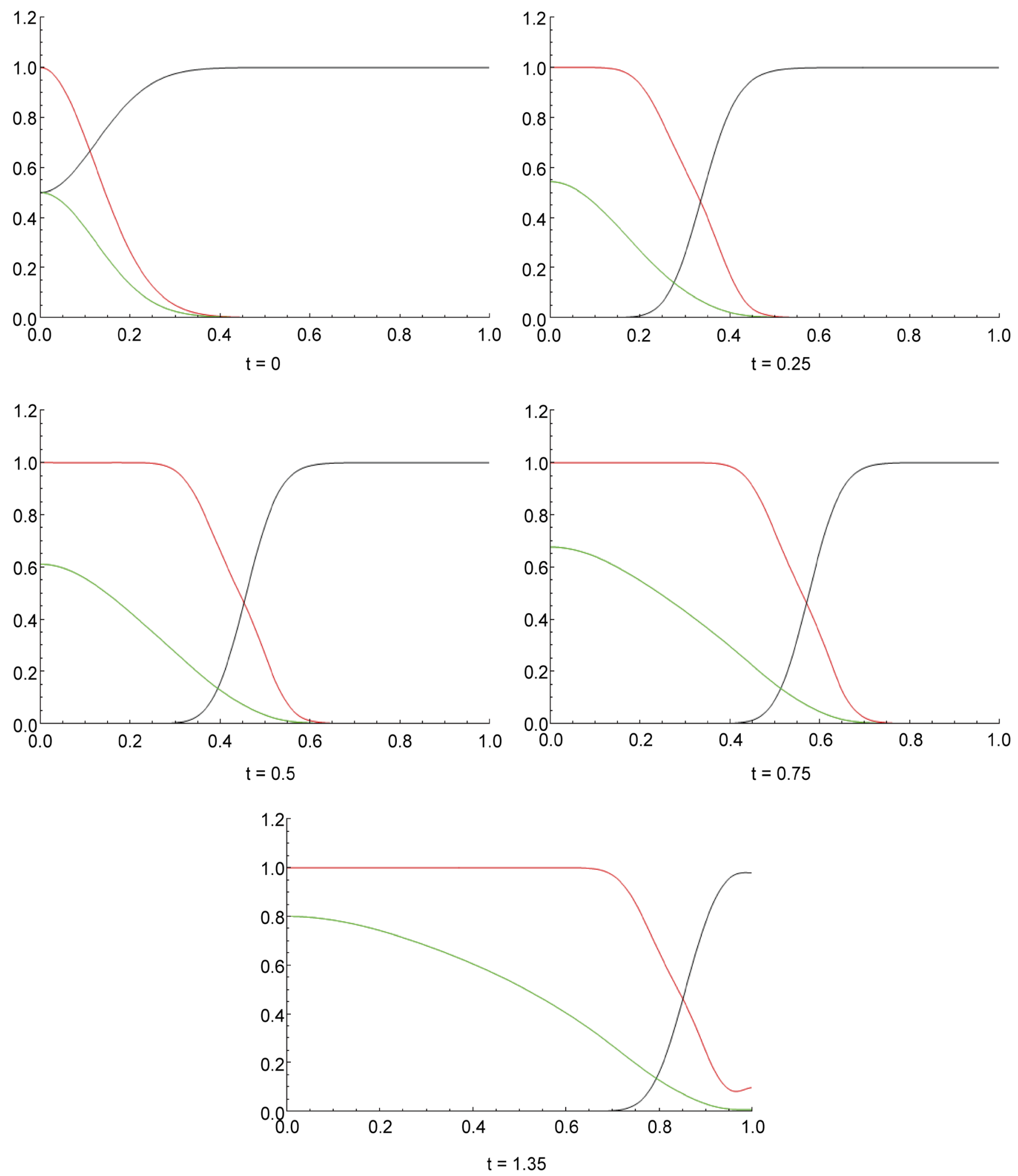

Figure 3. Tumour cell proliferation, migration, without ECM re-establishment, and interactions between the tumour and the surrounding tissue: The parameter values $d_{n}=0.001, d_{m}=0.001, \gamma=0.02, \eta=10, \mu_{1}=10, \mu_{2}=0, \alpha=0.1$ and $\beta=0.1, x_{n}=0$. Increasing $\mu_{1}$ more, it is observed that tumour cell density inside ECM become higher than in Figure 2. 

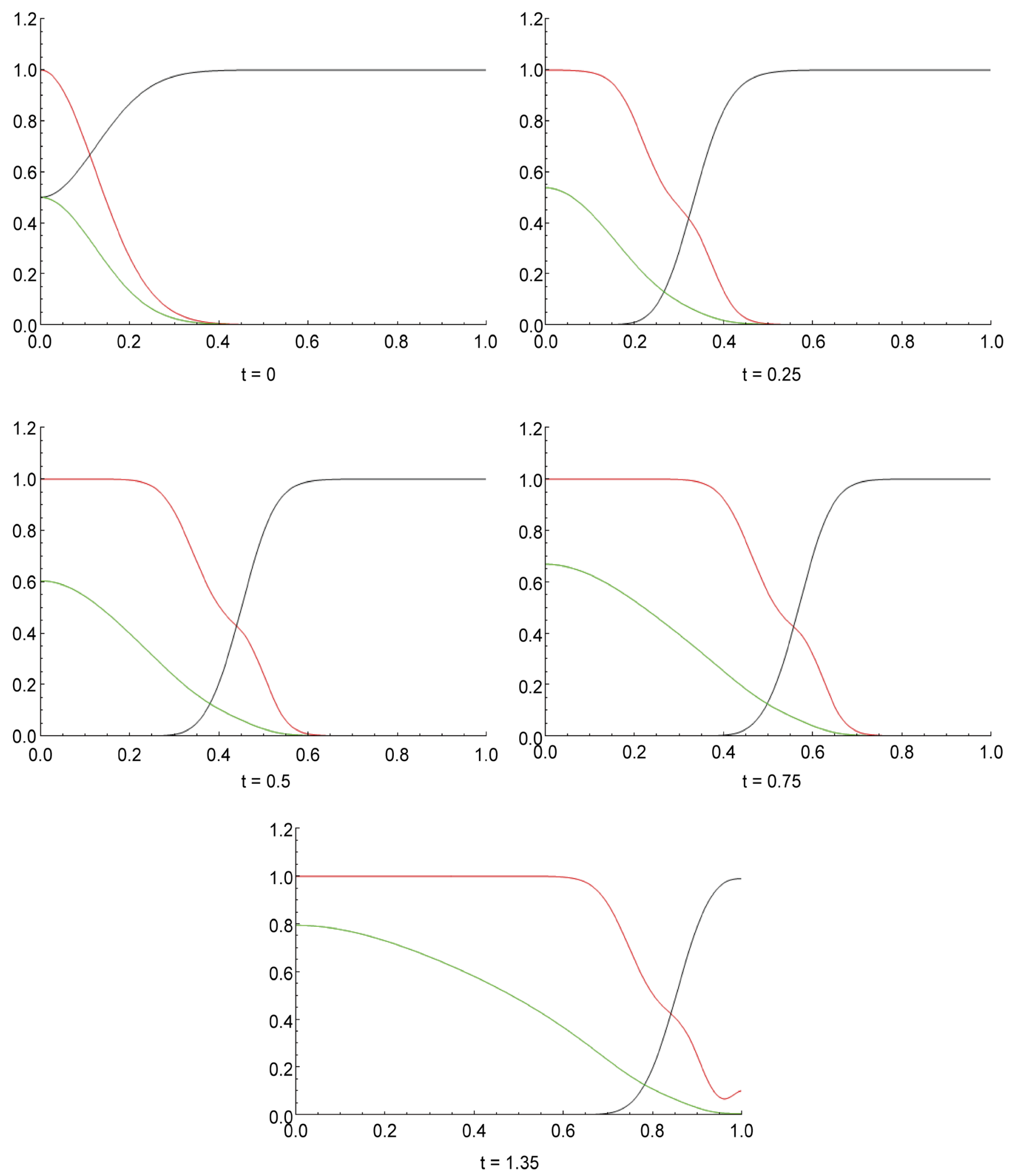

Figure 4. Tumour cell proliferation, migration, ECM re-establishment, and interactions between the tumour and the surrounding tissue: The parameter values $d_{n}=0.001, d_{m}=0.001, \gamma=0.02, \eta=10, \mu_{1}=5, \mu_{2}=0.001, \alpha=0.1$ and $\beta=0.1, x_{n}=0$. We take $\mu_{2}=0.001$ only and other parameters are same as in Figure 2. Then the tumour cell density is almost same as in Figure 2. 

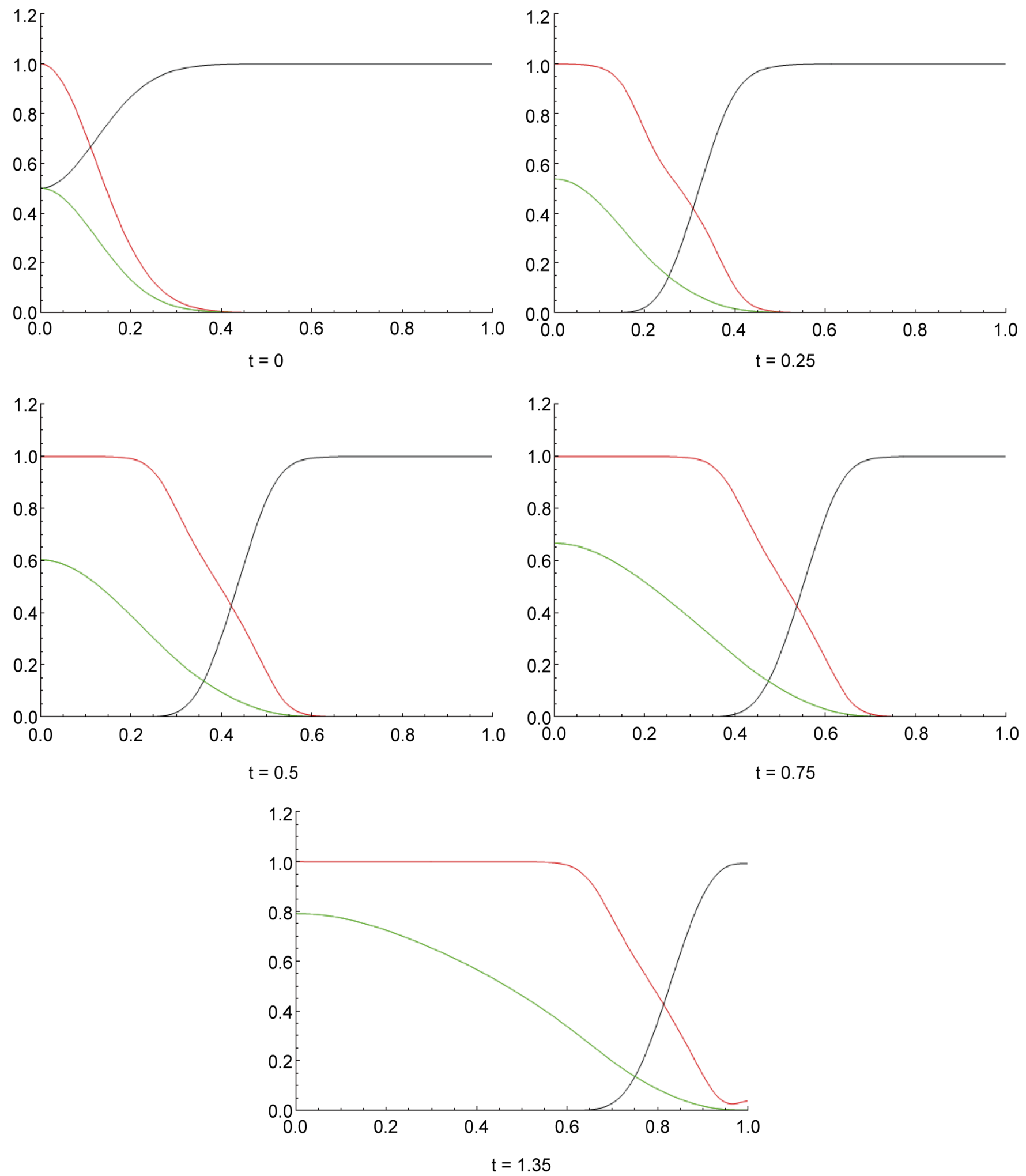

Figure 5. Tumour cell proliferation, migration, ECM re-establishment, and interactions between the tumour and the surrounding tissue: The parameter values $d_{n}=0.001, d_{m}=0.001, \gamma=0.02, \eta=10, \mu_{1}=5, \mu_{2}=1, \alpha=0.1$ and $\beta=0.1, x_{n}=0$. When taking $\mu_{2}=0.001$, compared with Figure 2 and Figure 4, it is clear that the invasive area of tumour cells inside ECM is much more limited. 

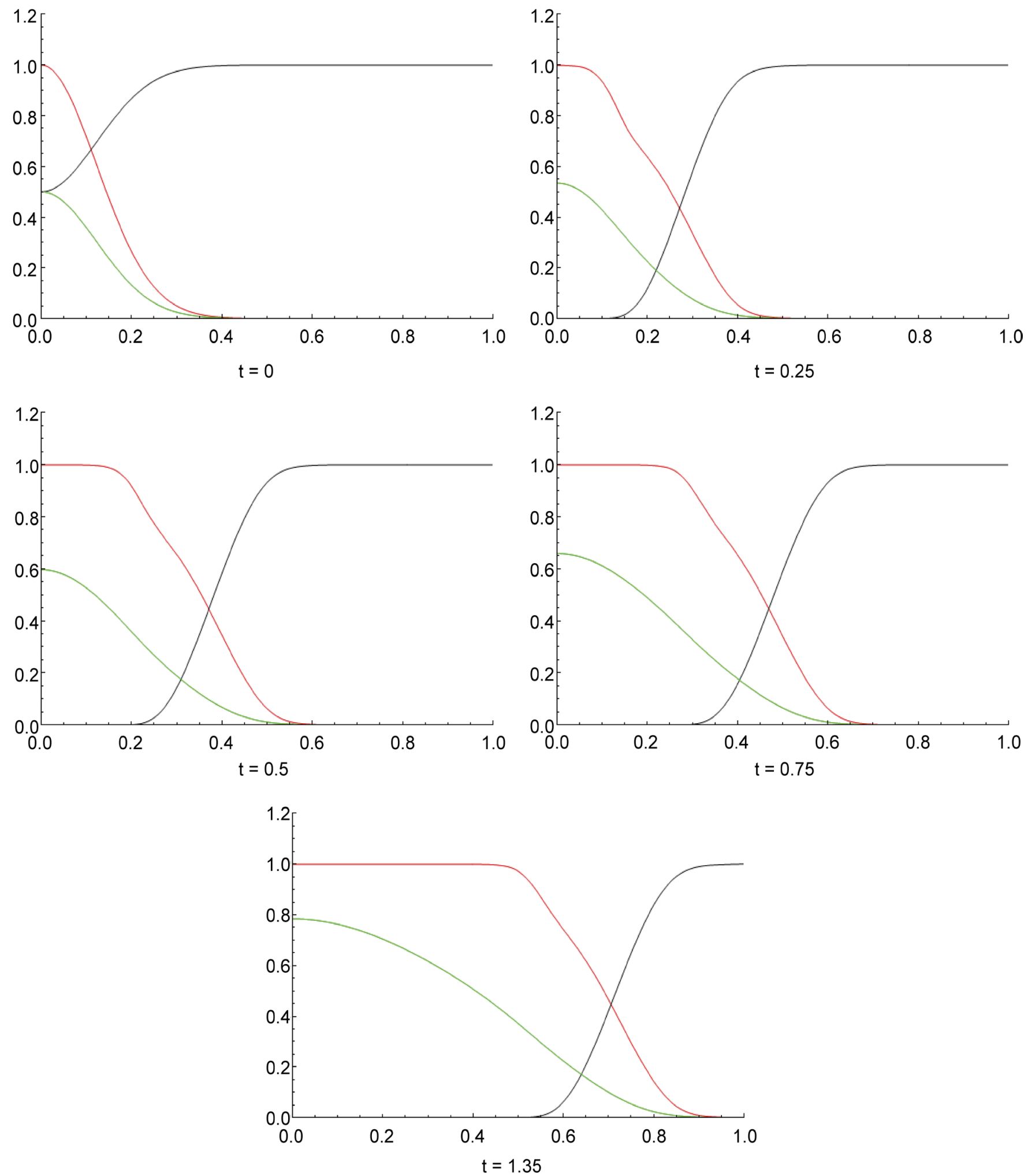

Figure 6. Tumour cell proliferation, migration, ECM re-establishment, and interactions between the tumour and the surrounding tissue: The parameter values $d_{n}=0.001, d_{m}=0.001, \gamma=0.02, \eta=10, \mu_{1}=5, \mu_{2}=5, \alpha=0.1$ and $\beta=0.1, x_{n}=0$. Compared with Figure 4 and Figure 5 , the reachable range of tumour cells is observed to be more suppressed by taking $\mu_{2}=5$. 

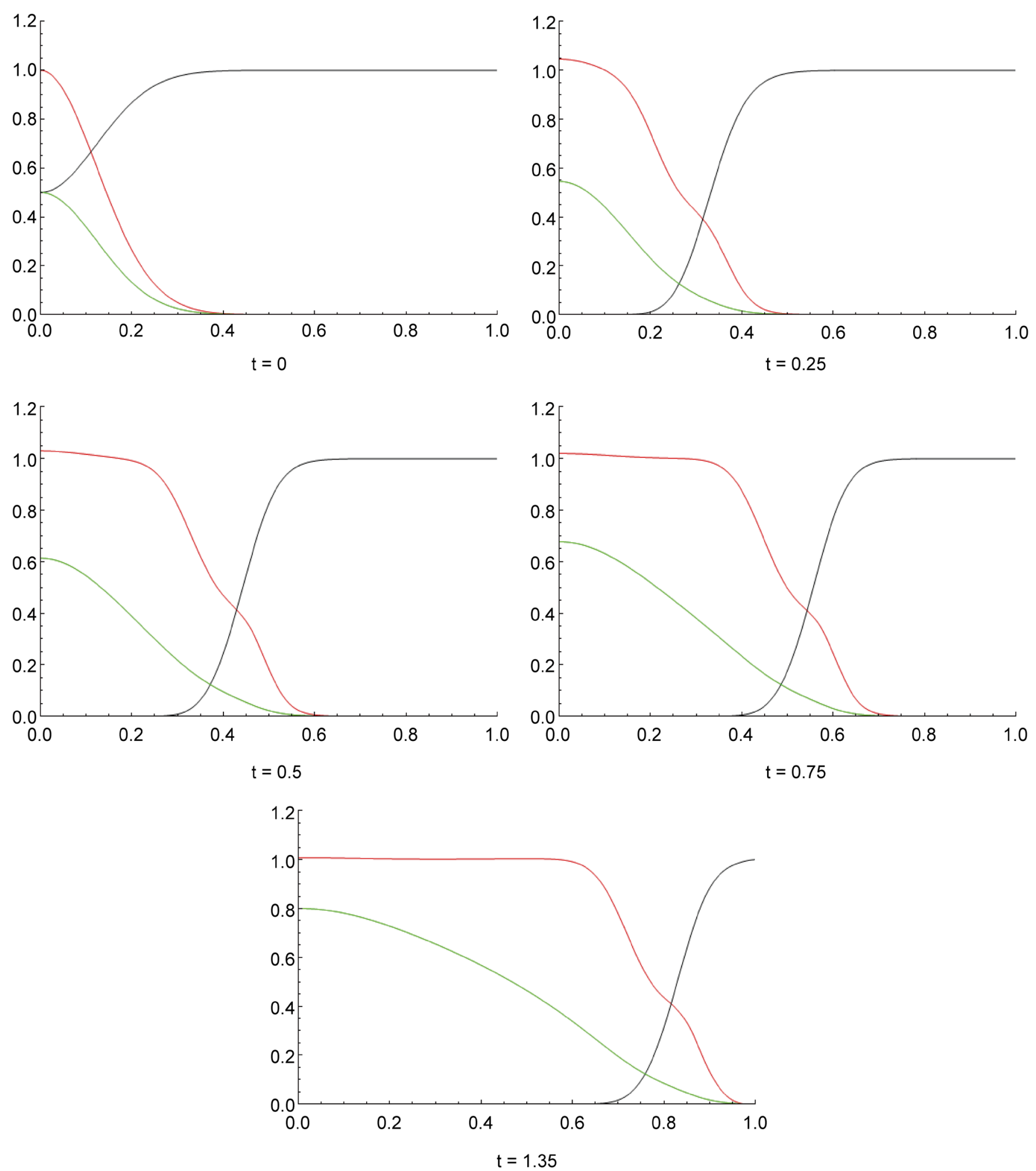

Figure 7. Tumour cell proliferation, chemotaxis, migration, ECM re-establishment, and interactions between the tumour and the surrounding tissue: The parameter values $d_{n}=0.001, d_{m}=0.001, \gamma=0.02, \eta=10, \mu_{1}=5, \mu_{2}=0, \alpha=0.1$ and $\beta=0.1, x_{n}=0.01$. By the effect of chemotaxis $\left(x_{n}=0.01\right)$, tumour cells are attracted by MDEs, the density is beyond 1 at $t=0.25 \sim 0.5$, eventually it converges to 1 and after that keeps it constantly. 


\section{Conclusions}

In order to obtain the global existence in time and asymptotic profile of solutions of a mathematical model of tumour invasion proposed by Chaplain and Lolas, we investigate nonlinear evolution equations with logistic term related to our mathematical models as an initial Neumann-boundary value problem. We could show the global existence in time of rigorous mathematical solutions to the initial boundary value problem for the model in arbitrary space dimension by using the energy inequalities. Applying the result to our model we show global existence in time of mathematical solutions of the model.

By Figures 1-7, it is recognized that our rigorous mathematical result of the existence and asymptotic behaviour of smooth solutions verifies our computer simulations and confirms the pattern form of each component of the model in the graphs respectively. Then we can gain the understanding of the process of tumour invasion more in details.

\section{Acknowledgements}

This work was supported in part by the Grants-in-Aid for Scientific Research (C) 19540200, 22540208, 25400148 and 16K05214 from Japan Society for the Promotion of Science.

\section{References}

[1] Chaplain, M.A.J. and Lolas, G. (2006) Mathematical Modeling of Cancer of Tissue: Dynamic Heterogeneity. Networks and Heterogeneous Media, 1, 399-439. http://dx.doi.org/10.3934/nhm.2006.1.399

[2] Anderson, A.R.A. and Chaplain, M.A.J. (2003) Mathematical Modelling of Tissue Invasion, In: Preziosi, L., Ed., Cancer Modelling and Simulation, Chapman Hall/CRC, 269-297.

[3] Kubo, A. and Kimura, K. (2014) Mathematical Analysis of Tumour Invasion with Proliferation Model and Simulations. WSEAS Transaction on Biology and Biomedicine, 11, 165173.

[4] Kubo, A. and Hoshino, H. (2015) Nonlinear Evolution Equation with Strong Dissipation and Proliferation, Current Trends in Analysis and Its Applications. Springer, Birkhauser, 233-241.

[5] Kubo, A. and Suzuki, T. (2004) Asymptotic Behavior of the Solution to a Parabolic ODE System Modeling Tumour Growth. Differential and Integral Equations, 17, 721-736.

[6] Kubo, A., Suzuki, T. and Hoshino, H. (2005) Asymptotic Behavior of the Solution to a Parabolic ODE System. Mathematical Sciences and Applications, 22, 121-135.

[7] Kubo, A. and Suzuki, T. (2007) Mathematical Models of Tumour Angiogenesis. Journal of Computational and Applied Mathematics, 204, 48-55. http://dx.doi.org/10.1016/j.cam.2006.04.027

[8] Kubo, A., Saito, N., Suzuki, T. and Hoshino, H. (2006) Mathematical Models of Tumour Angiogenesis and Simulations, Theory of Bio-Mathematics and Its Application. RIMS Kokyuroku, 1499, 135-146.

[9] Kubo, A. (2011) Nonlinear Evolution Equations Associated with Mathematical Models, Discrete and Continuous Dynamical Systems, Supplement, 881-890. 
[10] Dionne, P. (1962) Sur les problèmes de Cauchy hyperboliques bien posés. Journal d Analyse Mathematique, 10, 1-90. http://dx.doi.org/10.1007/BF02790303

[11] Andasari, V., Roper, R.T., Swat, M.H. and Chaplain, M.A.J. (2011) Integrating Intracellular Dynamics Using CompuCell3D and Bionetsolver: Applications to Multiscale Modelling of Cancer Cell Growth and Invasion. PLoS ONE, 7, e33726. http://dx.doi.org/10.1371/journal.pone.0033726

[12] Anderson, A.R.A. and Chaplain, M.A.J. (1998) Continuous and Discrete Mathematical Models of Tumour-Induced Angiogenesis. Bulletin of Mathematical Biology, 60, 857-899. http://dx.doi.org/10.1006/bulm.1998.0042

[13] Deakin, N.E. and Chaplain, M.A.J. (2013) Mathematical Modeling of Cancer Invasion: The Role of Membrane-Bound Matrix Metalloproteinases. Frontiers in Oncology, 3, 70. http://dx.doi.org/10.3389/fonc.2013.00070

[14] Hatami, F. and Ghaemi, M.B. (2013) Numerical Solution of Model of Cancer Invasion with Tissue. Applied Mathematics, 4, 1050-1058. http://dx.doi.org/10.4236/am.2013.47143

[15] Kim, Y. and Othmer, H.G. (2013) A Hybrid Model of Tumor-Stromal Interactions in Breast Cancer. Bulletin of Mathematical Biology, 75, 1304-1350. http://dx.doi.org/10.1007/s11538-012-9787-0

[16] Kolev, M. and Zubik-Kowal, B. (2011) Numerical Solutions for a Model of Tissue Invasion and Migration of Tumourcells. Computational and Mathematical Methods in Medicine, 2011, Article ID: 452320. http://dx.doi.org/10.1155/2011/452320

[17] Mahiddin, N. and Hashim, A. (2014) Approximate Analytical Solutions for Mathematical Model of Tumourinvasion and Metastasis Using Modified Adomian Decomposition and Homotopy Perturbation Methods. Journal of Applied Mathematics, 2014, Article ID: 654978. http://dx.doi.org/10.1155/2014/654978

[18] Märkl, C., Meral, G. and Surulescu, C. (2013) Mathematical Analysis and Numerical Simulations for a System Modeling Acid-Mediated Tumor Cell Invasion. International Journal of Analysis, 2013, Article ID: 878051.

[19] Orlando, P.A., Gatenby, R.A. and Brown, J.S. (2013) Tumor Evolution in Space: The Effects of Competition Colonization Tradeoffs on Tumor Invasion Dynamics. Frontiers in Oncology, 3, 45. http://dx.doi.org/10.3389/fonc.2013.00045

[20] Levine, H.A. and Sleeman, B.D. (1997) A System of Reaction and Diffusion Equations Arising in the Theory of Reinforced Random Walks. SIAM Journal on Applied Mathematics, 57, 683-730. http://dx.doi.org/10.1137/S0036139995291106

[21] Othmer, H.G. and Stevens, A. (1997) Aggregation, Blowup, and Collapse: The ABCs of Taxis in Reinforced Random Walks. SIAM Journal on Applied Mathematics, 57, 10441081. http://dx.doi.org/10.1137/S0036139995288976

[22] Chaplain, M.A.J., Lachowicz, M., Szymanska, Z. and Wrzosek, D. (2011) Mathematical Modeling of Cancer Invasion: The Importance of Cell-Cell Adhesion and Cell-Matrix Adhesion. Mathematical Models \& Methods in Applied Sciences, 21, 719-743. http://dx.doi.org/10.1142/S0218202511005192

[23] Sleeman, B.D. and Levine, H.A. (2001) Partial Differential Equations Chemotaxis and Angiogenesis. Mathematical Methods in the Applied Sciences, 24, 405-426. http://dx.doi.org/10.1002/mma.212

[24] Yang, Y. Chen, H. and Liu, W. (1997) On Existence and Non-Existence of Global Solutions to a System of Reaction-Diffusion Equations Modeling Chemotaxis. SIAM Journal on Applied Mathematics, 33, 763-785. http://dx.doi.org/10.1137/S0036141000337796 
Submit or recommend next manuscript to SCIRP and we will provide best service for you:

Accepting pre-submission inquiries through Email, Facebook, LinkedIn, Twitter, etc.

A wide selection of journals (inclusive of 9 subjects, more than 200 journals)

Providing 24-hour high-quality service

User-friendly online submission system

Fair and swift peer-review system

Efficient typesetting and proofreading procedure

Display of the result of downloads and visits, as well as the number of cited articles

Maximum dissemination of your research work

Submit your manuscript at: http://papersubmission.scirp.org/

Or contact apm@scirp.org 